\title{
A Cocktail Approach Toward Tunable Organic Afterglow Systems
}

Liangwei Ma, Qingyang Xu, Bingbing Ding, Zizhao Huang, Siyu Sun, Xiang Ma*, He Tian

L. Ma, Q. Xu, Dr. B. Ding, Z. Huang, S. Sun, Prof. X. Ma*, Prof. H. Tian

Key Laboratory for Advanced Materials and Feringa Nobel Prize Scientist Joint Research Center, Frontiers Science Center for Materiobiology and Dynamic Chemistry, School of Chemistry and Molecular Engineering, East China University of Science \& Technology, Shanghai 200237

E-mail: maxiang@ecust.edu.cn

Keywords: long-lived luminescence, energy transfer, cocktail approach, apparent lifetime

Abstract: In this work, a cocktail approach toward tunable organic long-lived luminescence materials in solid, solution, and gel states is proposed. The tunable long-lived luminescence $(\tau$ $>0.7 \mathrm{~s}$ ) is realized by controlling the energy transfer via manipulating the photo-induced isomerization of the energy acceptor (5). The afterglow can be regulated between blue and yellow emission upon irradiation of UV or visible light. And the "apparent lifetime" for the long-lived fluorescence is the same as the lifetime of the energy donor. The function is relying on the simple radiative energy transfer (reabsorption) between a long-lived phosphorescence and a highly efficient fluorescent isomer $(\mathbf{5 b})$, rather than the complicated communication between the excited state of the molecules such as Förster resonance energy transfer or Dexter energy transfer. The simple working principle endows this strategy with huge universality, flexibility, and operability. This work offers an extremely simple, feasible, and universal way to construct tunable afterglow materials in solid, solution, and gel states. 


\section{Introduction}

Metal-free room-temperature phosphorescence (RTP) materials, which are characterized by long-lived lifetime and triplet state involved radiative transition, have drawn overwhelming attention due to their potential in various fields like cell image, ${ }^{[1]}$ organic light-emitting diode, ${ }^{[2]}$ anti-counterfeiting, ${ }^{[3,4]}$ sensor, ${ }^{[5]}$ and so forth. $\left.{ }^{[6,} 7\right]$ However, it was difficult to construct efficient metal-free RTP materials, especially RTP materials with afterglow, because of their weak spin-orbit coupling (SOC), low intersystem crossing yield ( $\Phi_{\text {ISC }}$ ) of organic molecules, and susceptible nature of triplet state. ${ }^{[8,9]}$ In recent years, many efficient RTP materials ${ }^{[10]}$ with various lifetime and wavelength were obtained via some feasible strategies, like embedding into a matrix, ${ }^{[3,}$ ${ }^{11]}$ host-guest assembly, ${ }^{[1,12]}$ crystallization, ${ }^{[13,14]}$ heavy atom effect, and El-Sayed rule. ${ }^{[8,}$ ${ }^{15]}$ However, how to manipulate the phosphorescence wavelength was still a mystery because the phosphor with efficient phosphorescence emission needed a delicate molecular skeleton and design. Traditional design strategies on fluorochrome, ${ }^{[16]}$ like the push-pull system, seemed to be an efficient strategy to tune the wavelength of phosphorescence. ${ }^{[7,17]}$ However the modification on molecular skeleton often resulted in the phosphorescence silence of dyes. Manipulating the phosphorescence wavelength has become a big challenge.

Except for modifying the molecular skeleton via molecular engineering, harnessing the energy transfer between a phosphorescent energy donor and a fluorescent energy acceptor was an alternative way to obtain long-lived luminous materials. ${ }^{[18]}$ According to the mechanism of non-radiative energy transfer, the lifetime for the fluorescence of the energy acceptor could be prolonged to millisecond or even second level and the emission wavelength could also be shifted to long-wavelength region. ${ }^{[19]}$ However, it was difficult to construct a non-radiative efficient energy transfer system because energy reaction could happen only when the distance between the energy donor and acceptor 
was small enough (10 nm for Förster resonance energy transfer (FRET) energy transfer, and $1 \mathrm{~nm}$ for Dexter energy transfer) and the energy level was matched with each other. ${ }^{[20]}$ Similar to non-radiative energy transfer, the emission wavelength of the energy donor could also be shifted to the long-wavelength region via a radiative energy transfer process. While the lifetime for the energy donor and acceptor was keeping constant before and after the energy transfer process. But if the energy donor was a long-lived phosphor with decent afterglow, a long-lived fluorescence for the energy acceptor might be observed because the afterglow could work as an excitation source to continuously excite the energy acceptor after the excitation light was ceased. Moreover, the measured "lifetime" for the fluorescence, which was defined as "apparent lifetime", should identify with the energy donor (Equation S1-5). If this proposal could work, it would be a very easy strategy to construct long persistent luminescence materials with various colours because the radiative energy transfer process only requires the overlapping of emission and absorption spectra.

Photochromic dyes were a kind of important energy acceptors because of their distinct variations on absorption and emission spectra during the conversion between two isomers. Taking advantage of it, the energy transfer process could be manipulated by light. In 2011, Irie and his co-workers reported a unique diarylethene with a highly fluorescent closed-form and non-fluorescent open-form, which was contrary to transitional diarylethene derivatives. ${ }^{[21]}$ Upon the irradiation of UV light, the colorless 1 , 4-dioxane solution of the open-form (5a) diarylethene converted to yellow, and a new absorption peak around $456 \mathrm{~nm}$ was aroused (Scheme 1a). Further irradiated with visible light, the yellow solution could recover to the initial state. More importantly, the 1, 4dioxane solution of the closed-form (5b) shown strong fluorescence around $533 \mathrm{~nm}$ with an $87 \%$ fluorescence quantum yield, while no emission was recorded for the open-form 
(5a). The strong emission of the closed-ring isomer and non-fluorescent of the open-ring isomer made compound $\mathbf{5}$ be a perfect candidate for a tuneable energy acceptor.

In our recent work, we found a strong RTP material (1BBI-DMBA) with a $14.8 \%$ phosphorescence quantum yield (465 nm, Scheme 1a) and $122 \mathrm{~ms}$ lifetime. ${ }^{[14]}$ The cyan afterglow could be observed after the excitation source was turned off for $1.5 \mathrm{~s}$. Coincidently, the emission wavelength of 1BBI-DMBA was overlapped with the absorption spectra of $\mathbf{5 b}$ and orthogonal with 5a. Therefore, the cyan afterglow might be switched to yellow via UV light irradiation if the energy transfer process between $\mathbf{5 b}$ and 1BBI-DMBA could occur.

a)
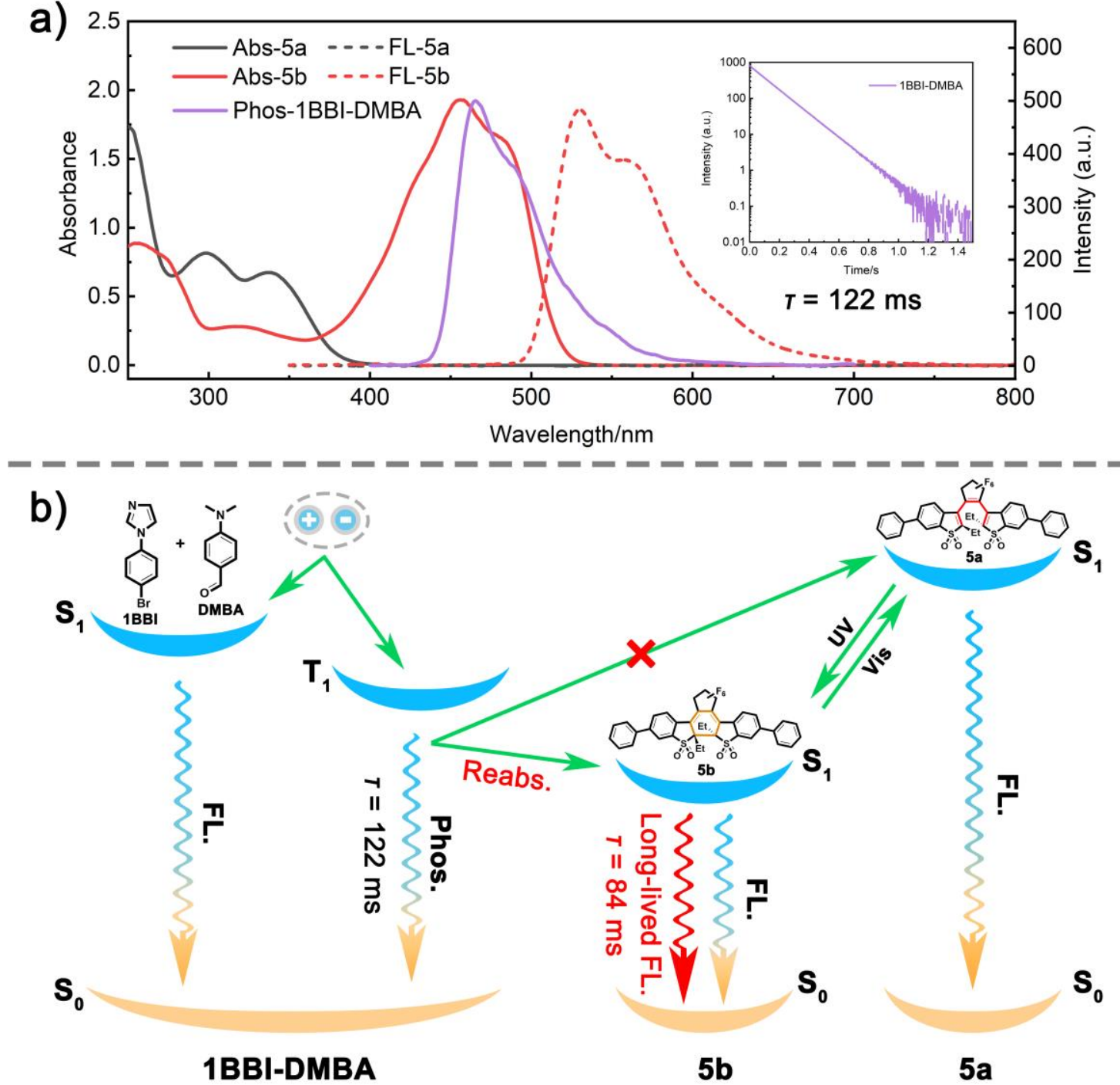

Figure 1. a) Absorption and emission spectra of $\mathbf{5 a}$ and $\mathbf{5 b}$ in 1,4-dioxane and the phosphorescence spectra of 1BBI-DMBA; Inset: lifetime decay spectra of 1BBI-DMBA at 470 $\mathrm{nm}$; b) Sketch for the working mechanism of the tunable afterglow based on the radiative energy transfer. 


\section{Result and Discussion}

1BBI (95 wt\%), DMBA (1 wt\%), and 5 ( $4 \mathrm{wt} \%)$ were mixed by dissolving them in methanol and evaporated under vacuum. Recent research showed that phosphorescence was extremely sensitive to impurity. ${ }^{[13,14,22]}$ With the assistant of trace ingredients, even nonluminous materials could exhibit strong phosphorescence with a long lifetime. To verify whether the presence of $\mathbf{5}$ in $\mathbf{1 B B I}$ will induce phosphorescence or alter the photophysical properties of 5, 5 (2.0 wt \%) was added to 1BBI (named 1BBI-5). With irradiation of the light at $365 \mathrm{~nm}$, two new absorption peaks around 461 and $493 \mathrm{~nm}$ were aroused for the absorption spectra of 1BBI-5 (Figure S1a), which was consistent with the absorption spectra of $\mathbf{5 b}$ in 1,4-dioxane. The variation of the absorption spectra indicated the occurrence of photochromism of 5 in the 1BBI matrix. 1BBI-5 showed a weak fluorescence around $538 \mathrm{~nm}$ (Figure S1a) in the initial state, which should be assigned to the trace of $\mathbf{5 b}$ in $\mathbf{1 B B I - 5}$. Further irradiating it with UV light, the emission intensity increased rapidly. The fluorescence should originate from $\mathbf{5 b}$ because the profile of the excitation and emission spectra were the same as $\mathbf{5 b}$ in 1,4-dioxane. These phenomena testified the occurrence of photochromism of 5 in the 1BBI matrix. And no phosphorescence emission was recorded for it no matter before or after UV light irradiation (Figure S1a), indicating that the addition of 5 in 1BBI would not arouse phosphorescence emission. To explore whether the mixture of 1BBI and DMBA (1BBIDMBA, $1 \mathrm{wt} \%$ ) could exhibit light responsiveness, the absorption and phosphorescence spectra of it were measured. As shown in Figure S1b, only a negligible variation was observed for both the absorption and emission spectra before and after UV light irradiation, which indicated the light stability of 1BBI-DMBA.

Identified with 1BBI-5, new absorption peaks around 462 and $495 \mathrm{~nm}$ for 1BBIDMBA-5 (4 wt\%) were aroused with continuous irradiation of UV light (Figure 1a). And the photo-stationary state (PSS) was reached after $40 \mathrm{~s}$ continuous irradiation 
because the absorbance at $462 \mathrm{~nm}$ reached the maximum. The variation of absorption spectra was assigned to the photo-induced isomerization of $\mathbf{5}$ because the profile of it was identified with $\mathbf{5 b}$ in 1, 4-dioxane (Scheme 1a) or 1BBI matrix (Figure S1a), and 1BBI or DMBA didn't show light responsiveness (Figure S1b). Similar to 1BBI-5, a weak fluorescence peak around $538 \mathrm{~nm}$ was recorded for 1BBI-DMBA-5 (4 wt\%) when excited with a light at $478 \mathrm{~nm}$ in the initial state (Figure 1b). The intensity was increased with the irradiation of UV light until the PPS state reached (40 s). Besides, the excitation spectrum of it was identified with the absorption spectrum of $\mathbf{5 b}$ (Figure S2), which also indicated the fluorescence originated from $\mathbf{5 b}$.

1BBI-DMBA-5 showed distinct phosphorescence emission around $470 \mathrm{~nm}$ in the initial state (Figure 1c), which was coincident with the phosphorescence emission of 1BBI-DMBA (Figure S1b). Identified with 1BBI-DMBA, the lifetime of the phosphorescence emission was measured to be $125.0 \mathrm{~ms}$ (Figure 1e), hinting that a cyan afterglow could be observed after the excitation source was turned off. Surprisingly, the intensity of the phosphorescence peak around $470 \mathrm{~nm}$ decreased, and a new peak around $538 \mathrm{~nm}$ increased gradually with the irradiation of UV light (Figure 1c). When the PPS state was reached, the emission peak around $538 \mathrm{~nm}$ became the major emission peak, and the cyan afterglow switched to yellow-green. Specifically, the Commission International de l'Eclairage (CIE) coordinate shifted from $(0.19,0.30)$ to $(0.31,0.46)$ with the irradiation of UV light (Figure S3). The new aroused phosphorescence peak around $570 \mathrm{~nm}$ should be assigned to the radiative transition from the $\mathrm{S}_{1}$ state of $\mathbf{5 b}$ because the profile of the aroused phosphorescence peak was identified with the fluorescence emission of $\mathbf{5 b}$. While the excitation spectra, monitored at $570 \mathrm{~nm}$, of 1BBI-DMBA-5 before and after UV light irradiation were identified with the excitation spectra of the phosphorescence of 1BBI-DMBA, rather than the absorption spectra of 5b (Figure 1d), indicating that the emission was original from the excited state of 1BBI- 
DMBA rather than the directly stimulated transition of $\mathbf{5 b}$. Besides, the phosphorescence emission spectra of 1BBI-DMBA didn't show obvious change under continuous UV light irradiation (Figure S1b). And 1BBI-5 didn't show phosphorescence emission with the absence of DMBA (Figure S1a). More importantly, the absorption spectrum of $\mathbf{5 b}$ was overlapped very well with the phosphorescence emission of 1BBI-DMBA. These phenomena testified to the occurrence of energy transfer between 1BBI-DMBA and 5b. Thereby, the newly aroused phosphorescence peak was speculated to be the delayed emission of $\mathbf{5 b}$, which is sensitized by the phosphorescence of 1BBI-DMBA.
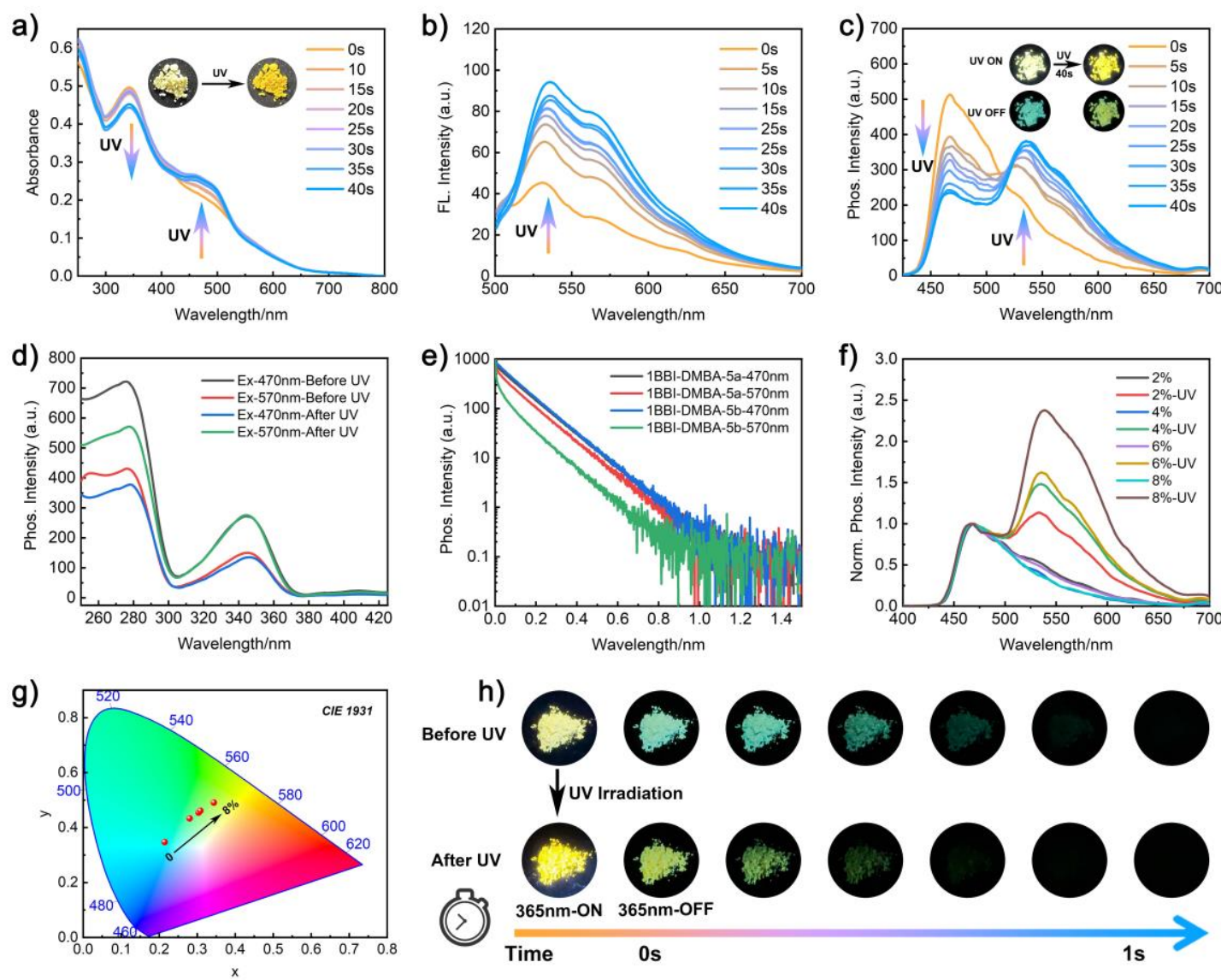

Figure 2. Variations of the absorption spectra (a), fluorescence spectra (b, $\lambda_{\mathrm{ex}}=478 \mathrm{~nm}$ ), phosphorescence spectra (c, delay time $=0.1 \mathrm{~ms}$, gate time $=2 \mathrm{~ms}, \lambda_{\mathrm{ex}}=345 \mathrm{~nm}$ ), excitation spectra of the phosphorescence emission at different wavelength (d), and life decay spectra at different wavelength (e, $\left.\lambda_{\mathrm{ex}}=345 \mathrm{~nm}\right)$ of 1BBI-DMBA-5 $(4 \mathrm{wt} \%)$ upon irradiation of $365 \mathrm{~nm}$ light; (f) Normalized phosphorescence emission spectra of 1BBI-DMBA-5 with different mass fraction before and after UV light irradiation; (g) CIE coordinate for the phosphorescence of 1BBI-DMBA-5 at PSS state with different mass fraction of 5; (h) Afterglow images of 1BBIDMBA-5 (8 wt \%) before and after UV irradiation. 
To further verify which mechanism of this energy transfer process was, the lifetime of the phosphorescence emission at different wavelengths was measured. As shown in Figure S4, the lifetimes of 1BBI-DMBA monitored at different wavelengths were consistent with each other and calculated to be $124.9 \mathrm{~ms}$. Identified with it, the lifetime of the phosphorescence of 1BBI-DMBA-5 at 470 and $570 \mathrm{~nm}$ were measured to be 125.0 and $108.0 \mathrm{~ms}$, respectively, in the initial state (Figure 1e, Table 1). After the cyan afterglow was switched to yellow-green by UV light, the lifetime of the phosphorescence at $570 \mathbf{n m}$, which was assigned to the delayed emission of $\mathbf{5 b}$, was measured to be 97.2 ms. While only a negligible decrease $(2.9 \mathrm{~ms})$ was observed for the lifetime of the phosphorescence emission at $470 \mathrm{~nm}$, which should result from the affection of the shorter lifetime for the phosphorescence emission at $570 \mathrm{~nm}$. Although the lifetime of the energy acceptor was increased from nanosecond to millisecond, the lifetime of the energy donor was kept constant. Thereby, the mechanism of the energy transfer process should be the reabsorption process, rather than FRET or Dexter energy transfer. The measured long apparent lifetime for the fluorescence confirmed our hypothesis about the radiative energy transfer with a long-lived emission energy donor. While the truth lifetime for it, which was measured to be 2.83 ns (Figure S5), should still be the same as the directly excited fluorescence (2.20 ns, Figure S5).

To further optimize its performance, different mass fractions of $\mathbf{5}$ were doped into 1BBI-DMBA. As illustrated in Figure 2a, all these crystalline (Figure S6) materials with different mass fractions of $\mathbf{5}(2,4,6$, or $8 \mathrm{wt} \%)$ exhibited similar phosphorescence spectra in the initial state. After switching to PSS state by UV light, all these materials showed a strong delayed emission around $538 \mathrm{~nm}$ with a decent apparent lifetime $(>80$ ms, Table 1, Figure S7), which hinted the energy transfer process occurred in the luminescence process for these materials. The intensity for the newly aroused emission peak was increased with the boosting of the mass fraction of $\mathbf{5}$, which might imply the 
increase of energy transfer efficiency. CIE coordinate also exhibited similar phenomena. As shown in Figure 2g, the CIE coordinate of the PSS state of these materials kept shifting to yellow-green with the increase of the mass fraction of 5. Specifically, a maximum variation was observed for the CIE coordinate of the phosphorescence when the mass fraction increased to $8 \mathrm{wt} \%$. And a clear yellow afterglow could also be observed for about $1 \mathrm{~s}$ when the excitation source was removed (Figure 2c).

Based on this strategy, long-live luminescent material could also be constructed in the solution state, which was a big challenge for RTP material. ${ }^{[23]}$ In previous work, our group has developed an ultra-long RTP material (P3), which exhibited a phosphorescence emission at $427 \mathrm{~nm}$ with an impressive $0.54 \mathrm{~s}$ lifetime and $15.4 \%$ phosphorescence quantum yield, by copolymerizing benzoic acid and acrylamide. ${ }^{[3]}$ After dispersing in $N, N$-Dimethylformamide (DMF) $(1 \mathrm{mg} / \mathrm{mL})$, the suspension still could exhibit long-lived phosphorescence emission $(\tau=0.74 \mathrm{~s})$ at $427 \mathrm{~nm}$ when excited at $280 \mathrm{~nm}$ (Figure S8). The emission of $\mathbf{P 3}$ was well overlapped with the absorption spectra of $\mathbf{5 b}$ in the DMF solution (Figure S9). Thereby, the afterglow of P3 might be regulated by light after mixed $\mathbf{5}$ and $\mathbf{P 3}$ in DMF. 

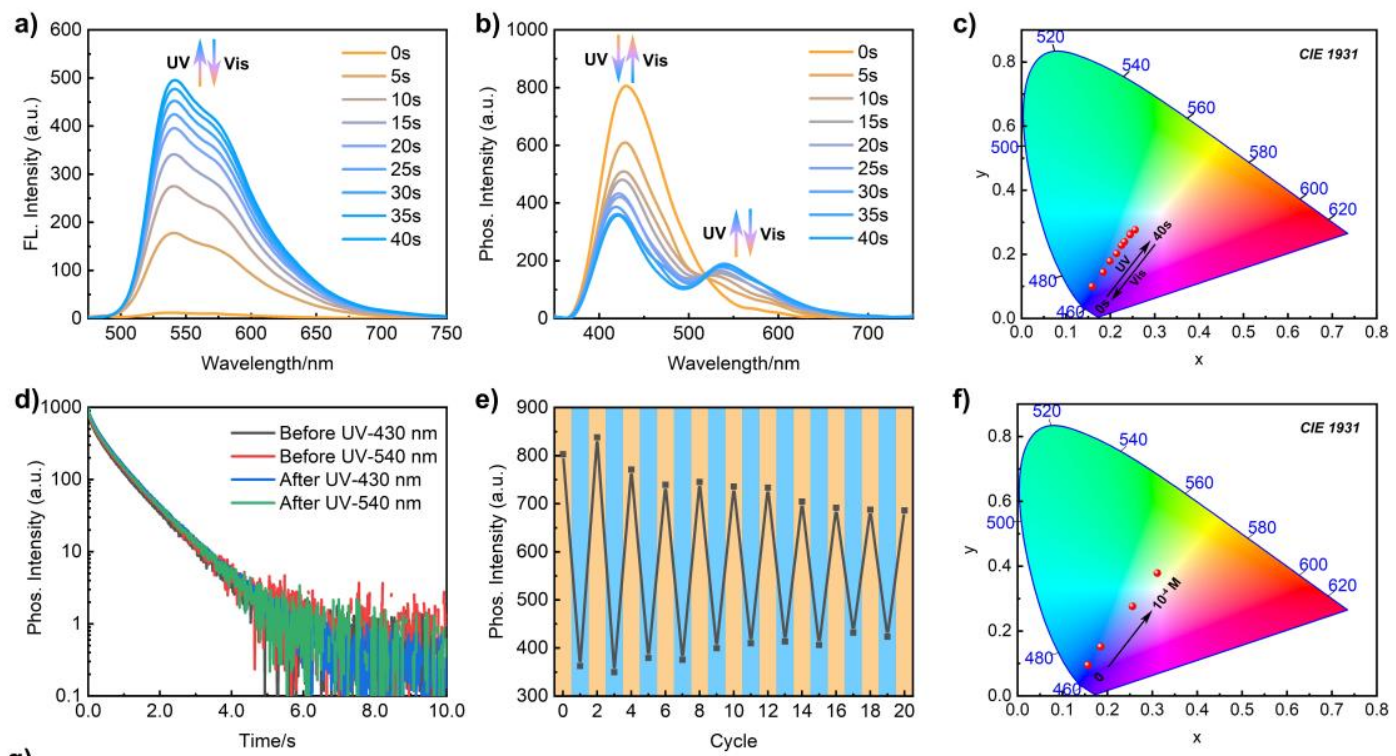

g)

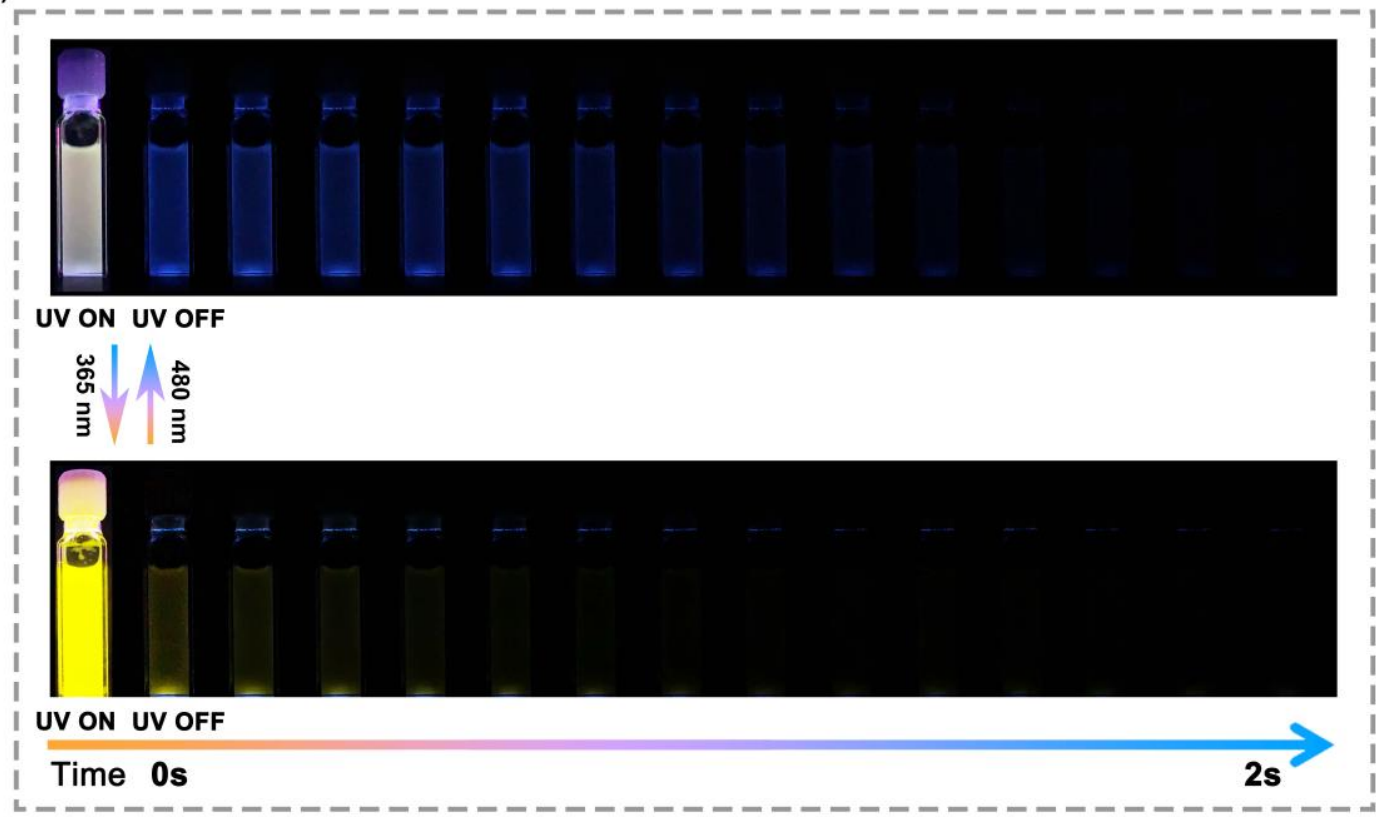

Figure 3. Fluorescence (a), phosphorescence (b, delay time $=0.1 \mathrm{~ms}$, gate time $=2 \mathrm{~ms}, \lambda_{\mathrm{ex}}=$ $280 \mathrm{~nm}$ ), and CIE coordinate (c) spectra of the DMF solution of P3-5 $\left(5.0 \times 10^{-5} \mathrm{M}\right)$ upon continues irradiation of UV light; (d) lifetime decay spectra of the DMF solution of P3-5 $\left(5.0 \times 10^{-5} \mathrm{M}\right)$ before and after UV light irradiation; (e) Variation for the phosphorescence emission intensity at $430 \mathrm{~nm}$ upon alternant irradiation of light at 365 and $480 \mathrm{~nm}$; (f) CIE coordinates for the phosphorescence emission of P3-5 with different concentration of 5 at PSS state; (g) Afterglow images for the DMF solution of P3-5 $\left(1.0 \times 10^{-4} \mathrm{M}\right)$ upon alternant irradiation of light at 365 and $480 \mathrm{~nm}$.

Identified with the DMF solution of 5, a strong fluorescence at $540 \mathrm{~nm}$, which was assigned to the emission of $\mathbf{5 b}$, was aroused for the mixture of $\mathbf{5}$ and $\mathbf{P 3}$ in DMF upon continuous irradiation of $365 \mathrm{~nm}$ light (Figure 3a). And the excitation spectra could well 
overlap with the absorption spectra of $\mathbf{5 b}$ (Figure S10). While the delayed emission at $427 \mathrm{~nm}$, which was assigned to the phosphorescence emission of $\mathbf{P 3}$, decreased gradually and a new emission peak at $540 \mathrm{~nm}$ was aroused. Similar to 1BBI-DMBA-5, the profile of the new aroused peak was the same as the fluorescence of $\mathbf{5 b}$, indicated that the emission was the radiative transition from the $S_{1}$ state of $\mathbf{5 b}$. And the excitation spectrum of the newly aroused delayed emission peak was the same as the phosphorescence emission of P3 (Figure S11), exhibiting that both the delayed emission at 427 and 540 $\mathrm{nm}$ originated from the same excitation process. Thereby, the new aroused peak should result from the $\mathbf{P 3}$ to $\mathbf{5 b}$ energy transfer process. To further testify it, the photophysical properties of the mixture $\mathbf{P 3}$ or $\mathbf{5}$ in DMF were measured. As illustrated in Figure S12, no obvious delayed emission was recorded for the DMF solution of $\mathbf{5}$ before or after UV light irradiation. And the phosphorescence emission intensity was kept constant before and after UV light irradiation for the mixture of $\mathbf{P 3}$ in DMF. These phenomena indicated the delayed emission at $540 \mathrm{~nm}$ resulted from the energy transfer process. Moreover, all the lifetimes for the delayed emission at 430 and $540 \mathrm{~nm}$ were measured to be about 0.7 s before and after UV light irradiation, which confirmed the radiative energy transfer mechanism and our hypothesis (Equation S1-5). The CIE coordinate of the delayed emission could shift from $(0.16,0.10)$ to $(0.26,0.28)$ when the PSS state was arrived, demonstrated the feasibility of our strategy on manipulating afterglow. Benefiting from the reversible isomerization and excellent fatigue resistance of $\mathbf{5}$, the afterglow could reversibly convert between two states (Figure 3g, 3e). And the switch could repeat for more than 10 cycles without distinct fading. Similar phenomena could also be observed when decreased or increased the concentration of 5 (Figure S13). It should be noticed that the concentration of $\mathbf{5}$ would not affect the lifetime of delayed emission, which further confirmed our hypothesis (Figure S14). While the CIE coordinate for the delayed emission at PSS state was shifted from blue to the yellow region with the increase of the 
concentration of 5 (Figure 3f). Specifically, the CIE coordinate could be reversibly switched between $(0.16,0.10)$ and $(0.31,0.38)$ when the concentration of 5 increased to $1.0 \times 10^{-4} \mathrm{M}$.

Organogel was a kind of important soft material, which was easy to be shaped to the desired pattern by simple annealing. ${ }^{[24]}$ Among various kinds of organogel, DOG was an efficient and easy obtained material, which could format transparent gel after dispersing in a solvent. ${ }^{[25]}$ Taking advantage of it, we could easily obtain organogel with tuneable afterglow by simply adding DOG into the DMF solution of P3-5. As shown in Figure 4a, a colorless gel was formed after cooling down the mixture of DOG (10 $\mathrm{mg} / \mathrm{ml}), \mathbf{P 3}(10 \mathrm{mg} / \mathrm{ml})$, and $\mathbf{5}\left(1.0 \times 10^{-4} \mathrm{M}\right)$ in DMF $(1.5 \mathrm{~mL})$ to room temperature. And the gel could be converted to a solution state after heating. Similar to the DMF solution of $\mathbf{5}$, the photochromism of compound $\mathbf{5}$ could also occur in gel or solution state when irradiated with UV or Visible light. The gel and the solution could be converted between colorless and yellow upon the irradiation of light. More importantly, a clear blue afterglow for the gel in the initial state could be observed for about $2 \mathrm{~s}$ after the excitation source $(254 \mathrm{~nm})$ was ceased. Upon irradiation of UV light, compound 5a isomerized to 5b gradually, accompanied with the blue to the yellow conversion of the afterglow. Identified with the solution or the solid, the yellow afterglow was assigned to the longlived emission from $\mathbf{5 b}$, which was excited by the afterglow of $\mathbf{P 3}$. Benefitting from the photochromic properties of $\mathbf{5}$, the afterglow could be reversibly switched between blue and yellow upon irradiation of UV or visible light. 

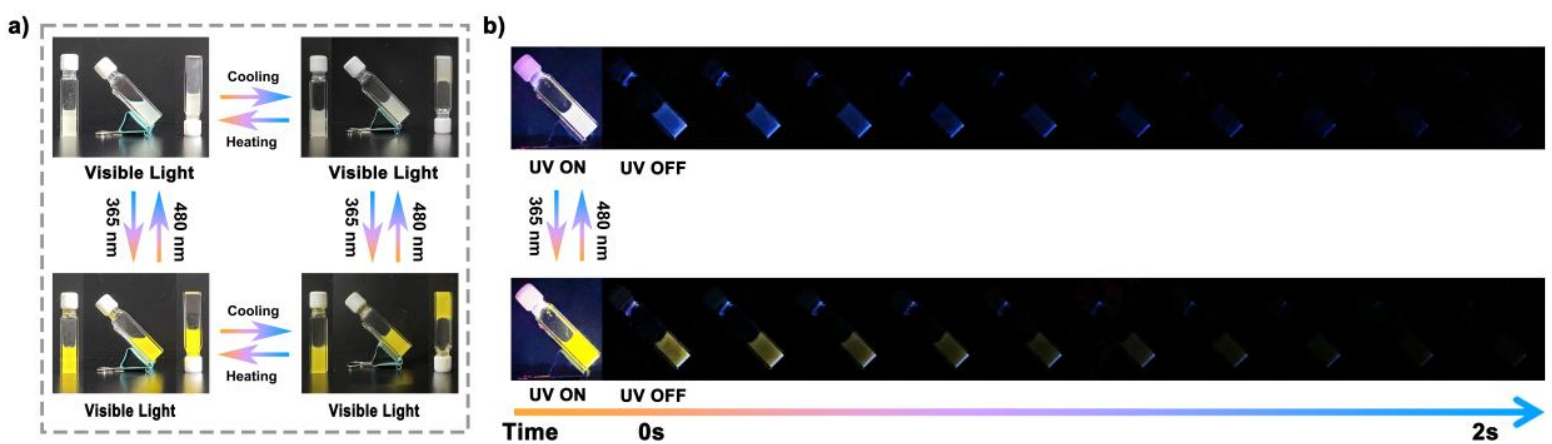

Figure 4. (a) Photograph for the DMF solution of P3-5-DOG before and after heating upon irradiation of UV or visible light irradiation; (b) Afterglow images of the gel of P3-5-DOG upon alternant irradiation of light at 365 and $480 \mathrm{~nm}$.

\section{Conclusion}

In conclusion, a universal, feasible, and simple cocktail approach toward tuneable afterglow systems in solid, solution, or gel state was proposed in this research. A series of long-lived fluorescence $(\tau>0.7 \mathrm{~s})$ materials were obtained via the radiative energy transfer between RTP materials and highly efficient fluorophores. The afterglow of these RTP materials could be reversibly switched between blue and yellow. Benefitting from the long lifetime of RTP materials, the fluorescence could be observed for several seconds after the excitation source was removed. The simple radiative energy transfer mechanism only requires the overlapping of the phosphorescence spectrum of the energy donor and the absorption spectrum of the energy acceptor, which provides this strategy with universal, feasible, and simple features on fabricating various kinds of long-live emission materials.

\section{Supporting Information}

Supporting Information is available from the Wiley Online Library or from the author.

\section{Acknowledgements}

We gratefully acknowledge the financial support from the National Natural Science Foundation of China (21788102, 22125803, 22020102006, and 21871083), project support by the Shanghai 
Municipal Science and Technology Major Project (Grant No.2018SHZDZX03), Program of Shanghai Academic/Technology Research Leader (20XD1421300), 'Shu Guang' project supported by Shanghai Municipal Education Commission and Shanghai Education Development Foundation (19SG26), the Innovation Program of Shanghai Municipal Education Commission(2017 01-07-00-02-E00010), and the Fundamental Research Funds for the Central Universities.

Received: ((will be filled in by the editorial staff))

Revised: ((will be filled in by the editorial staff)) Published online: ((will be filled in by the editorial staff))

\section{References}

[1] J. Wang, Z. Huang, X. Ma, H. Tian, Angew. Chem. Int. Ed. 2020, 59, 9928.

[2] R. Kabe, N. Notsuka, K. Yoshida, C. Adachi, Adv. Mater. 2016, 28, 655.

[3] X. Ma, C. Xu, J. Wang, H. Tian, Angew. Chem. Int. Ed. 2018, 57, 10854.

[4] H. Thomas, D. L. Pastoetter, M. Gmelch, T. Achenbach, A. Schlogl, M. Louis, X. Feng, S. Reineke, Adv. Mater. 2020, 32, 2000880.

[5] X. Q. Liu, K. Zhang, J. F. Gao, Y. Z. Chen, C. H. Tung, L. Z. Wu, Angew. Chem. Int. Ed. 2020, 59, 23456.

[6] S. Sun, L. Ma, J. Wang, X. Ma, H. Tian, Natl. Sci. Rev. 2021, DOI: 10.1093/nsr/nwab085; Z. Y. Zhang, W. W. Xu, W. S. Xu, J. Niu, X. H. Sun, Y. Liu, Angew. Chem. Int. Ed. 2020, 59, 18748; H. Shi, L. Zou, K. Huang, H. Wang, C. Sun, S. Wang, H. Ma, Y. He, J. Wang, H. Yu, W. Yao, Z. An, Q. Zhao, W. Huang, ACS Appl. Mater. Interfaces 2019, $11,18103$.

[7] L. Ma, S. Sun, B. Ding, X. Ma, H. Tian, Adv. Funct. Mater. 2021, 31, 2010659.

[8] N. J. Turro, The Benjamin/Cummings Publishing Company 1978. 
[9] X. Ma, J. Wang, H. Tian, Acc. Chem. Res. 2019, 52, 738; Kenry, C. Chen, B. Liu, Nat. Commun. 2019, 10, 2111; L. Bian, H. Ma, W. Ye, A. Lv, H. Wang, W. Jia, L. Gu, H. Shi, Z. An, W. Huang, Sci. China Chem. 2020, 63, 1443.

[10] T. Zhang, X. Ma, H. Wu, L. Zhu, Y. Zhao, H. Tian, Angew. Chem. Int. Ed. 2020, 59, 11206.

[11] Y. Su, S. Z. F. Phua, Y. Li, X. Zhou, D. Jana, G. Liu, W. Q. Lim, W. K. Ong, C. Yang, Y. Zhao, Sci. Adv. 2018, 4, eaas9732; H. Wu, W. Chi, Z. Chen, G. Liu, L. Gu, A. K. Bindra, G. Yang, X. Liu, Y. Zhao, Adv. Funct. Mater. 2018, 29, 1807243; H. Chen, X. Yao, X. Ma, H. Tian, Adv. Optical Mater. 2016, 4, 1397.

[12] Z. Y. Zhang, Y. Liu, Chem. Sci. 2019, 10, 7773; Z. Y. Zhang, Y. Chen, Y. Liu, Angew. Chem. Int. Ed. 2019, 58, 6028.

[13] C. Chen, Z. Chi, K. C. Chong, A. S. Batsanov, Z. Yang, Z. Mao, Z. Yang, B. Liu, Nat. Mater. 2020, 20, 175.

[14] B. Ding, L. Ma, Z. Huang, X. Ma, H. Tian, Sci. Adv. 2021, 7, eabf9668.

[15] M. S. Kwon, D. Lee, S. Seo, J. Jung, J. Kim, Angew. Chem. Int. Ed . 2014, 53, 11177.

[16] P. Liu, B. Li, J. Zheng, Q. Liang, C. Wu, L. Huang, P. Zhang, Y. Jia, S. Wang, Sens. Actuators B Chem. 2021, 329, 129147.

[17] M. Louis, H. Thomas, M. Gmelch, A. Haft, F. Fries, S. Reineke, Adv. Mater. 2019, 31, 1807887.

[18] Y. Ning, J. Yang, H. Si, H. Wu, X. Zheng, A. Qin, B. Z. Tang, Sci. China Chem. 2021, 64, 739.

[19] H. Gui, Z. Huang, Z. Yuan, X. Ma, CCS Chem. 2021, 481; S. Kuila, S. J. George, Angew. Chem. Int. Ed. 2020, 59, 9393; X. Tao, Z. Liao, Y. Zhang, F. Fu, M. Hao, Y. Song, E. Song, Chin. Chem. Lett. 2021, 32, 791. 
[20] C. Weng, N. Fan, T. Xu, H. Chen, Z. Li, Y. Li, H. Tan, Q. Fu, M. Ding, Chin. Chem. Lett. 2020, 31, 1490; Q. Ling, T. Cheng, S. Tan, J. Huang, L. Xu, Chin. Chem. Lett. 2020, 31, 2884.

[21] K. Uno, H. Niikura, M. Morimoto, Y. Ishibashi, H. Miyasaka, M. Irie, J. Am. Chem. Soc. 2011, 133, 13558.

[22] B. Chen, W. Huang, H. Su, H. Miao, X. Zhang, G. Zhang, Angew. Chem. Int. Ed. 2020, $59,10023$.

[23] S. Sun, J. Wang, L. Ma, X. Ma, H. Tian, Angew. Chem. Int. Ed. 2021. DOI: 10.1002/anie.202107323.

[24] L. Zhang, T. Wang, Z. Shen, M. Liu, Adv. Mater. 2016, 28, 1044; Y. Sun, Y. Zhang, Y. Song, Y. Liu, X. Zhang, Dyes Pigm. 2021, 193, 109546; W. J. Qu, H. H. Yang, J. P. Hu, P. Qin, X. X. Zhao, Q. Lin, H. Yao, Y. M. Zhang, T. B. Wei, Dyes Pigm. 2021, 186, 108949; J. Yuan, X. Dong, B. Zhang, Q. Zhou, S. Lu, Q. Wang, Y. Liao, Y. Yang, H. Wang, Dyes Pigm. 2020, $181,108506$.

[25] X. Zhu, Y. Jiang, D. Yang, L. Zhang, Y. Li, M. Liu, Chem. Sci. 2019, 10, 3873. 
A universal, feasible, and simple cocktail approach toward dynamic tuneable afterglow systems base on a simple radiative energy transfer mechanism is present. The afterglow $(\tau>0.7 \mathrm{~s})$ can be reversibly converted between blue and yellow in solid, solution, and gel state.

\section{A Cocktail Approach Toward Tunable Organic Afterglow Systems}

Liangwei Ma, Qingyang Xu, Bingbing Ding, Zizhao Huang, Siyu Sun, Xiang Ma*, He Tian

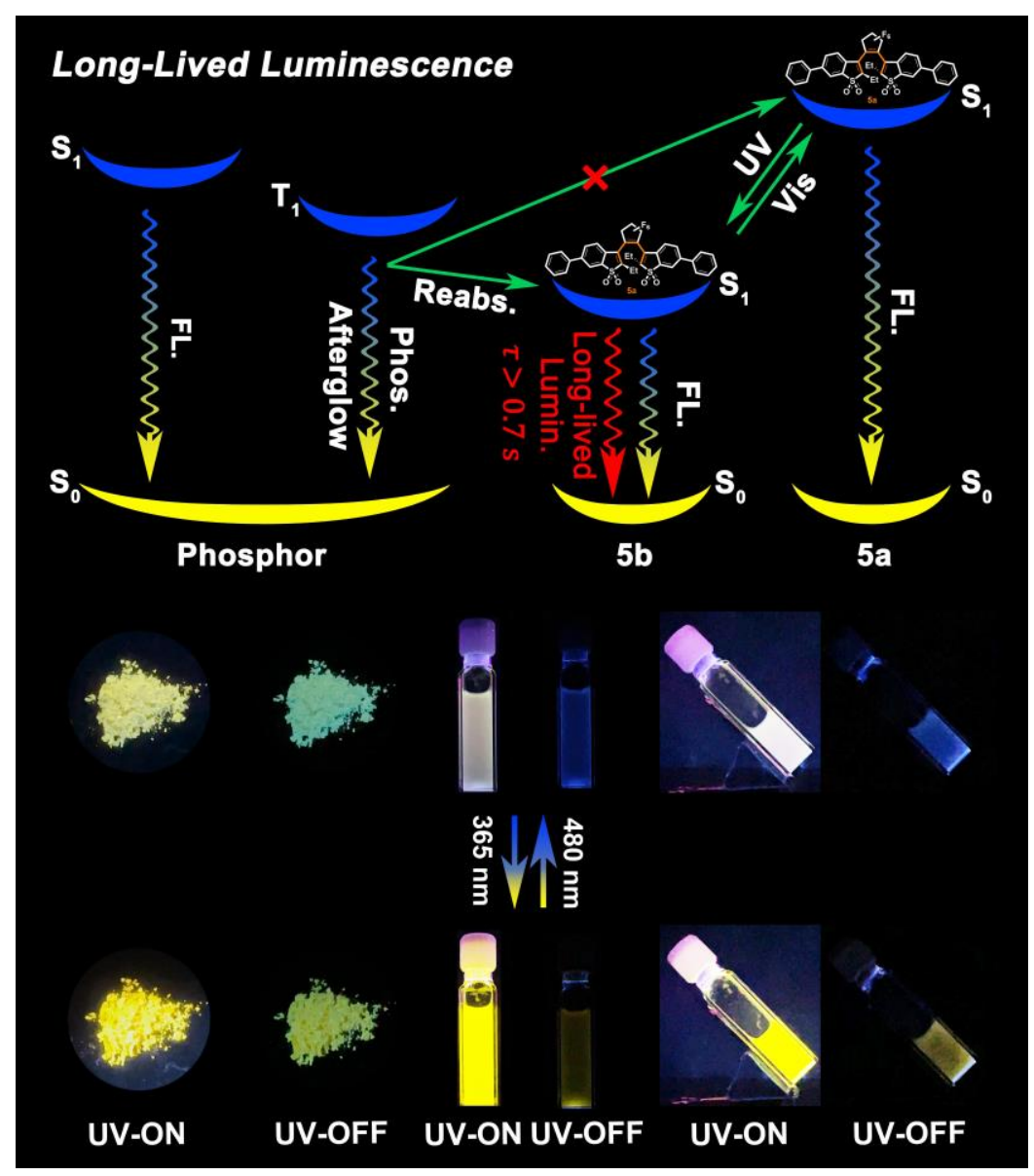




\section{Supporting Information}

\section{A Cocktail Approach Toward Tunable Organic Afterglow Systems}

Liangwei Ma, Qingyang Xu, Bingbing Ding, Zizhao Huang, Siyu Sun, Xiang Ma*, He Tian

General. ${ }^{1} \mathrm{H}$ NMR spectra were measured on a Bruker AV-400 spectrometer. The UV-Vis absorption spectra were obtained by a Shimadzu-2600 spectrophotometer. Emission spectra were recorded on an Agilent Cary Eclipse spectrophotometer. Fluorescence lifetimes were measured by Edinburgh Instruments Fluorescence Spectrometer (FLS1000). All the reagents were obtained from Adamas commercially.

For the long-lived phosphor (energy donor), the relationship between the emission intensity (I) and the time after the excitation source was ceased $(t)$ could express as:

$I=f(t)$

When $t=\tau(\tau$ : the lifetime of the phosphor):

$I_{\tau}=f(\tau)=\frac{f(0)}{e}$

For the fluorophore (energy acceptor), the relationship between the emission intensity $\left(I^{\prime}\right)$ and the time after the excitation source was ceased $(t)$ could express as:

$I^{\prime}=\Phi^{\prime} f(t)$

Where $\Phi$ ' was the Luminescence quantum yield of the fluorophore.

When $t=\tau^{\prime}\left(\tau^{\prime}\right.$ : measure lifetime of the fluorophore):

$\Phi^{\prime} f\left(\tau^{\prime}\right)=I_{(\tau \prime)}^{\prime}=\frac{I_{(0)}^{\prime}}{e}=\frac{\Phi^{\prime} f(0)}{e}=\Phi^{\prime} f(\tau)$ 
And $\Phi^{\prime} \neq 0, f(\tau) \neq 0, f\left(\tau^{\prime}\right) \neq 0$

Therefore:

$\tau^{\prime}=\tau$
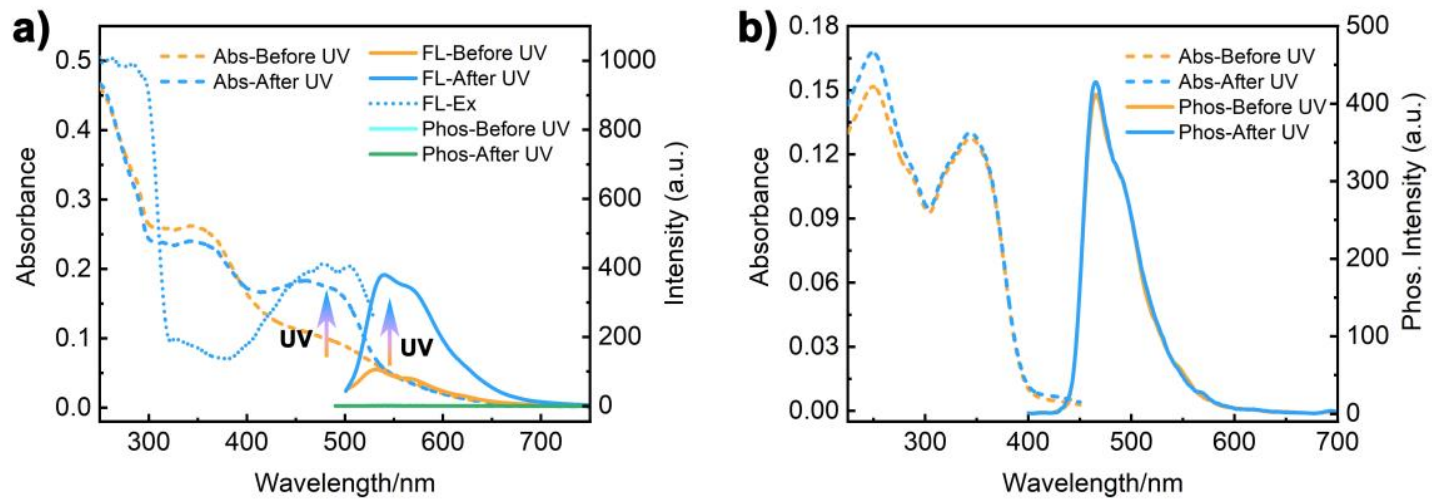

Figure S1. (a) Absorption, fluorescence, excitation, and phosphorescence spectra change of 1BBI-5 before and after UV light irradiation; $\lambda_{\mathrm{ex}}=478 \mathrm{~nm}$; (b) Absorption and phosphorescence spectra change of 1BBI-DMBA before and after UV light irradiation; $\lambda_{\mathrm{ex}}=$ $345 \mathrm{~nm}$.

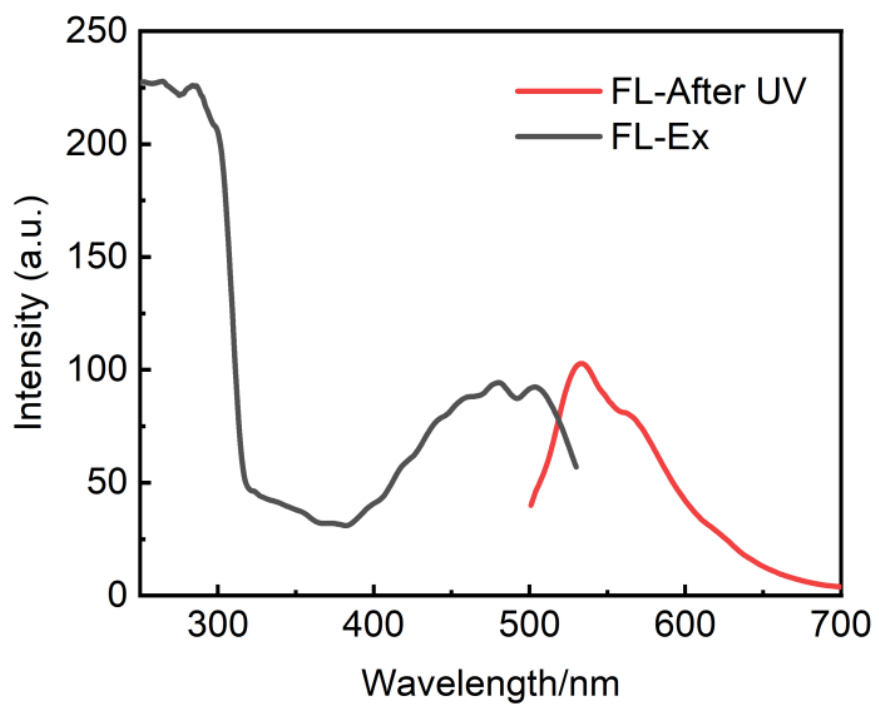

Figure S2. Excitation and emission spectra of 1BBI-DMBA-5 (4 wt \%) after UV light irradiation. 


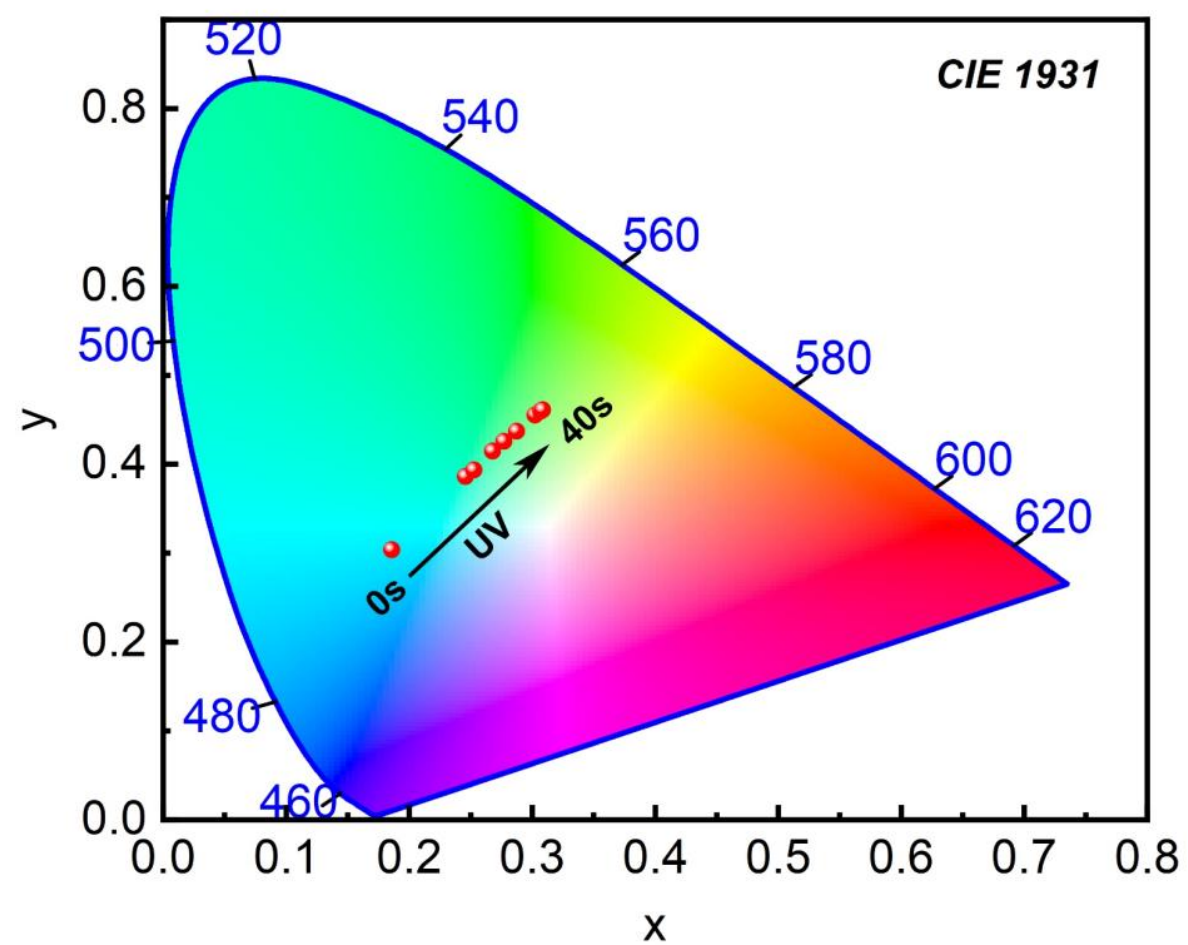

Figure S3. CIE coordinate changes for the phosphorescence of 1BBI-DMBA-5 (4 wt $\%)$ upon irradiation of $365 \mathrm{~nm}$ light.

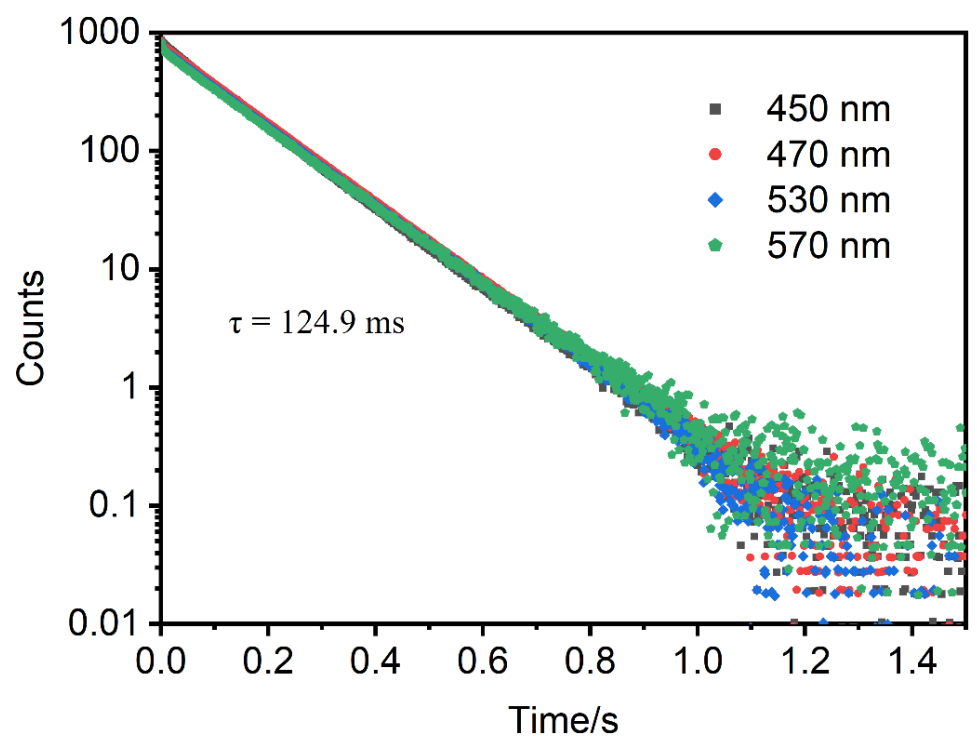

Figure S4. Lifetime decay spectra of 1BBI-DMBA at different wavelengths. 


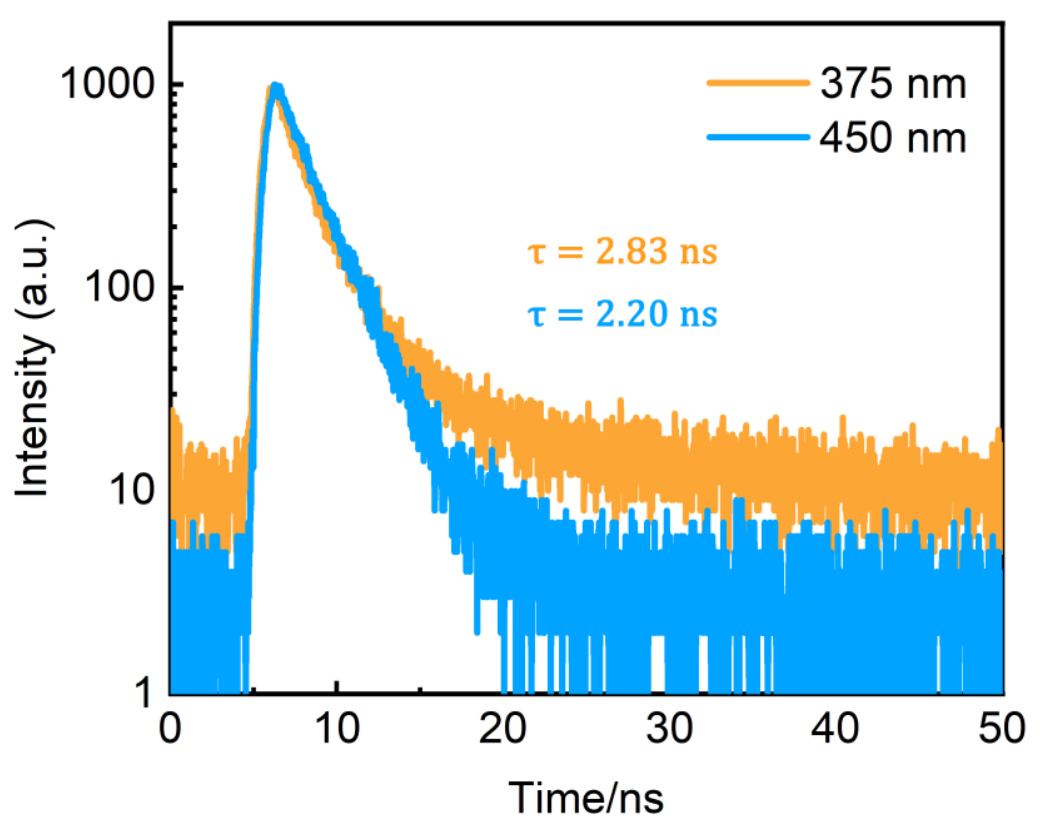

Figure S5. Lifetime decay spectra of the fluorescence of 1BBI-DMBA-5 (4 wt $\%$ ) at $570 \mathrm{~nm}$ after UV light excited at 375 and $450 \mathrm{~nm}$, respectively.

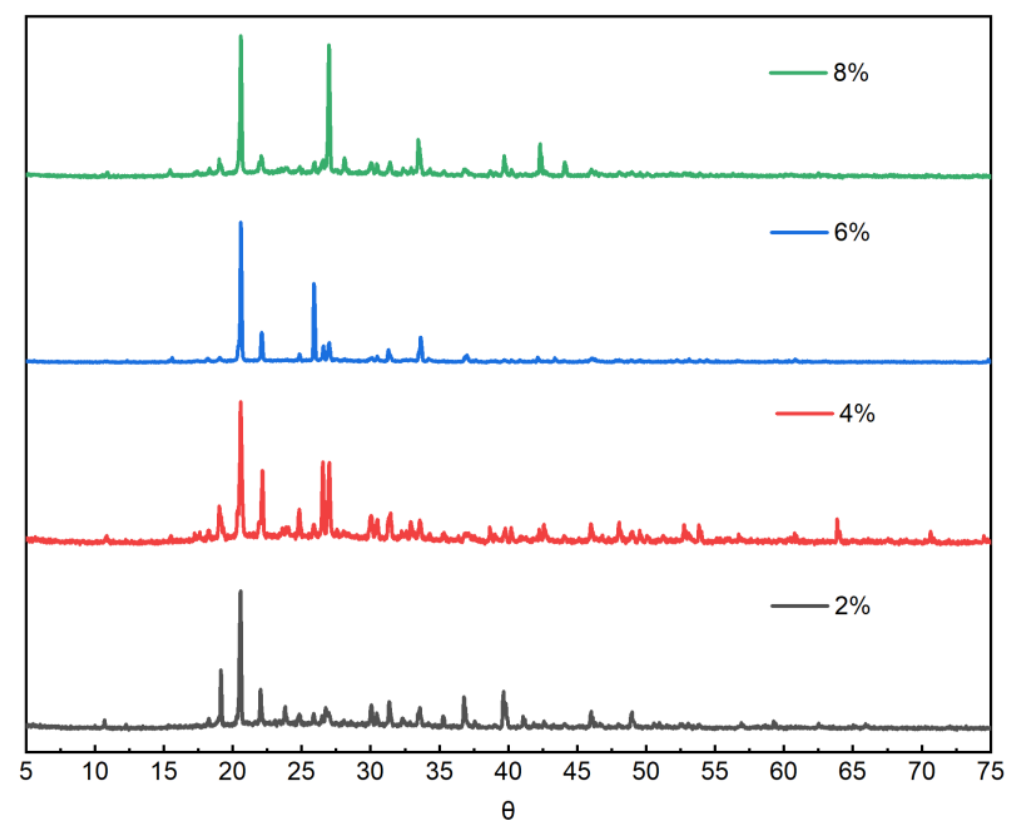

Figure S6. Powder X-ray diffraction (XRD) spectra of 1BBI-DMBA with different mass fractions of $\mathbf{5}$. 

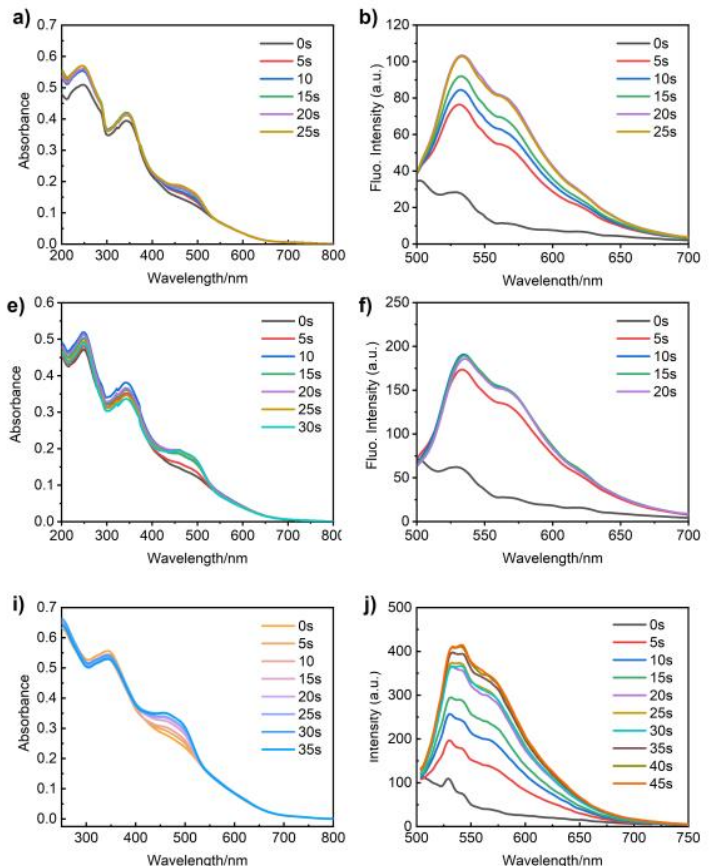
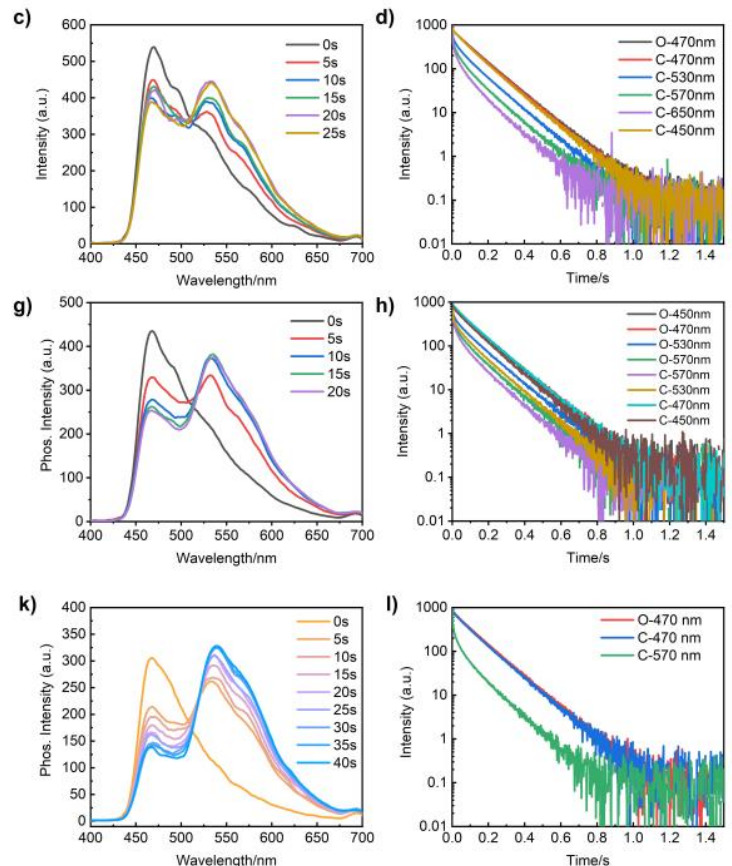

Figure S7. Absorption (a, e, and i), fluorescence (b, f, and j), phosphorescence (e, g, and k), lifetime decay spectra at difference wavelength ( $\mathrm{d}, \mathrm{h}$, and $\mathrm{l})$, and CIE spectra of 1BBI-DMBA with the presence of different ratio of $5(2 \%, 6 \%$, and $8 \%$ respectively).

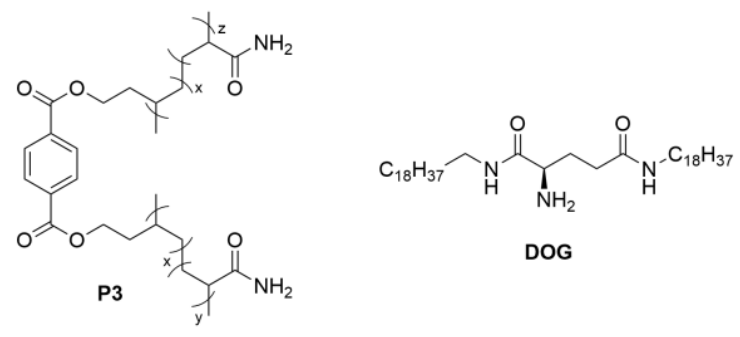

Scheme S1. Chemical structure of P3 and DOG. 

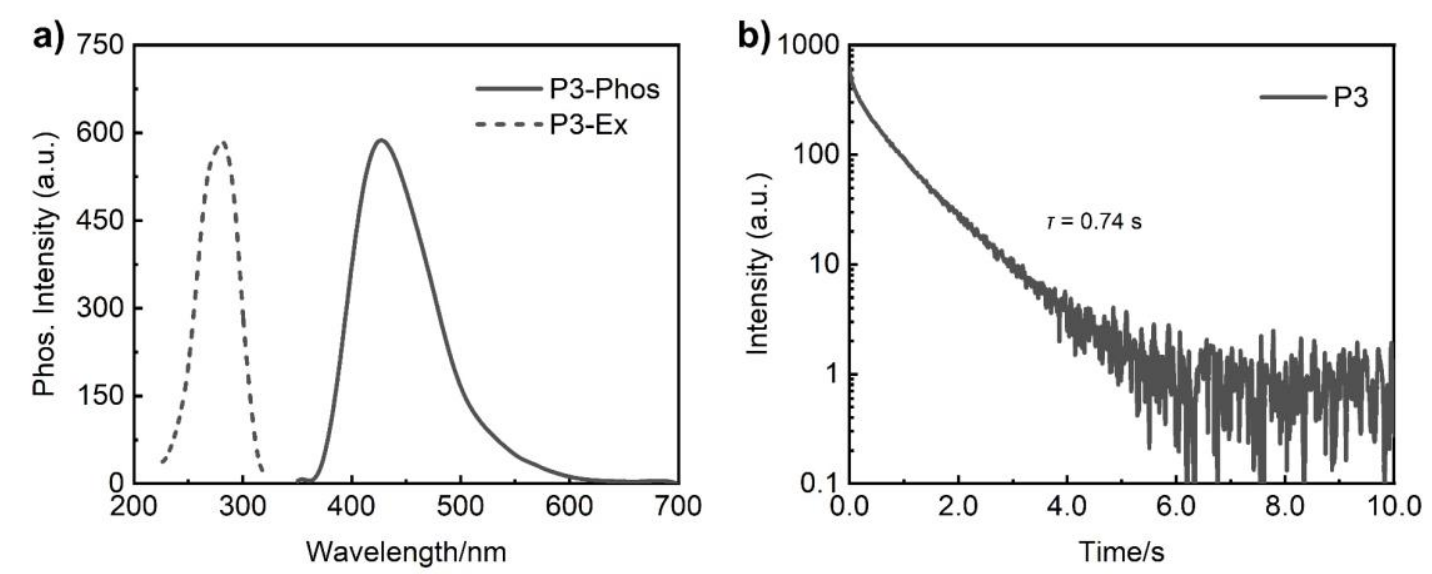

Figure S8. Excitation (a), phosphorescence (a), and lifetime decay spectra of P3 in DMF.

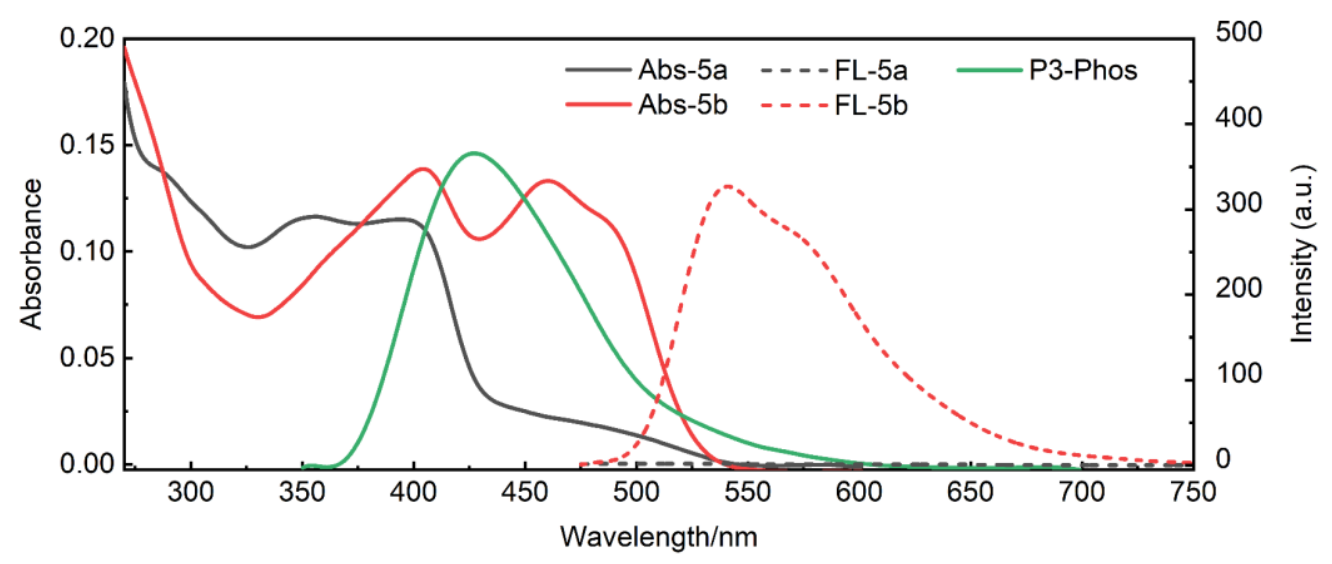

Figure S9. Absorption (solid line) and emission (dash line) spectra for the DMF solution of 5 before (gray line) and after (red line) UV light irradiation; Phosphorescence spectra of P3 in DMF. 


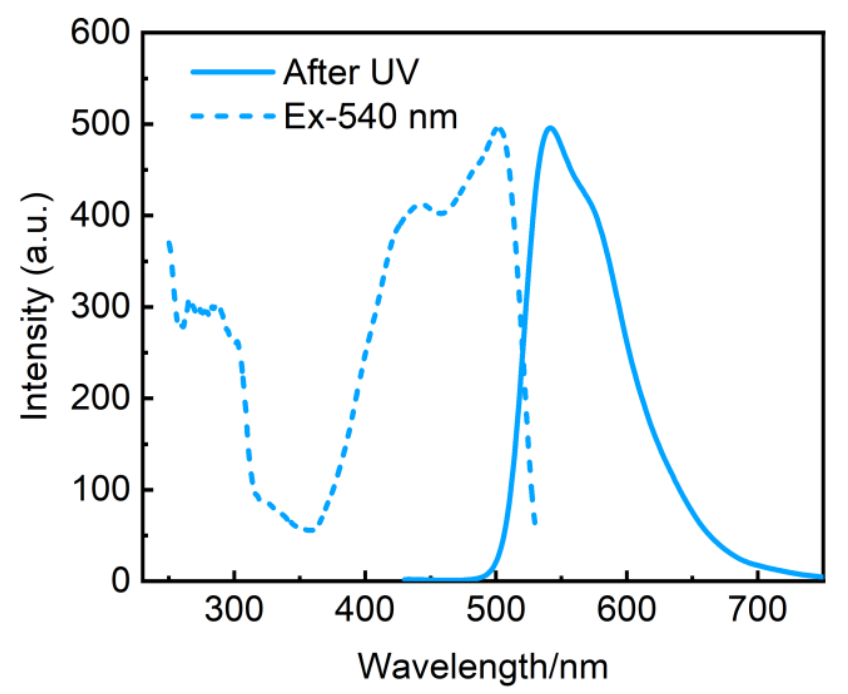

Figure S10. Emission and excitation spectra for the DMF solution of $\mathbf{5}$ after irradiation of UV light.

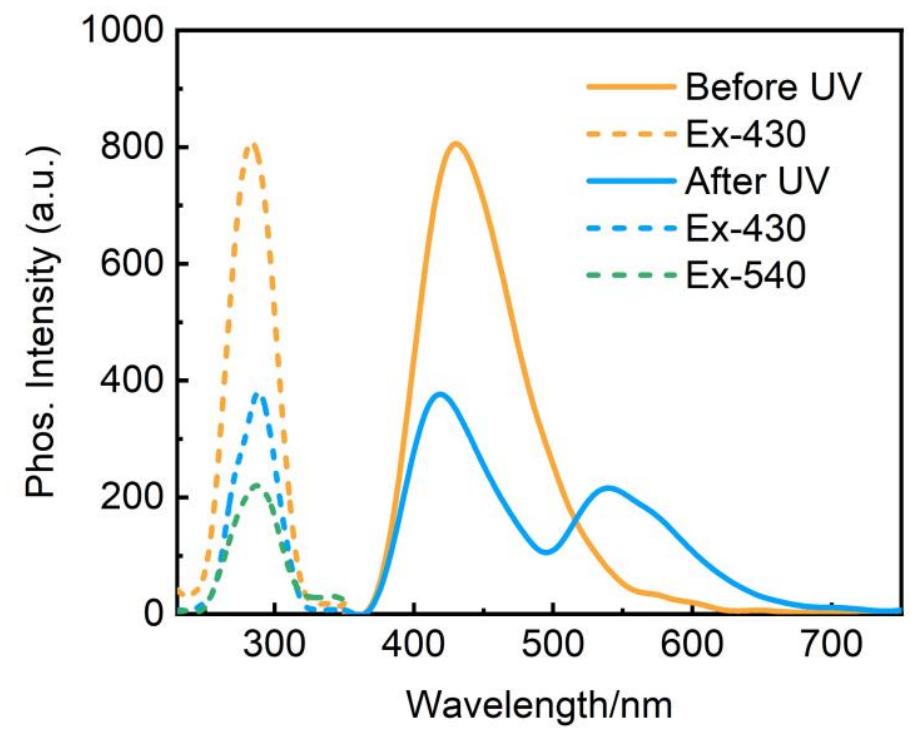

Figure S11. Excitation and phosphorescence spectra of the DMF solution of P3-5 $\left(5.0 \times 10^{-5} \mathrm{M}\right)$ before and after UV light irradiation. 


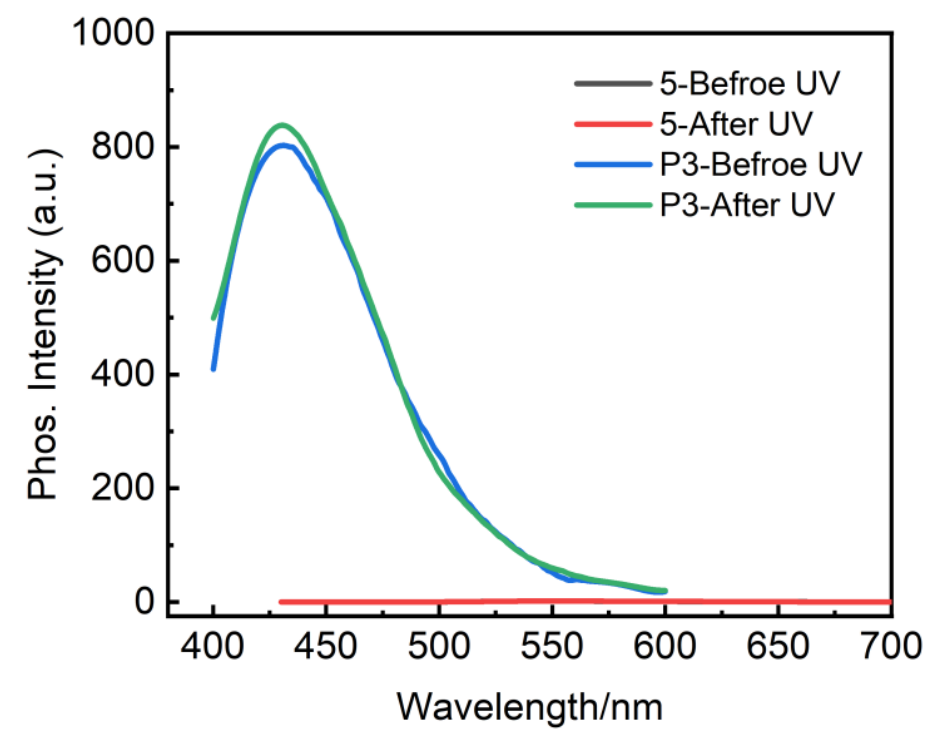

Figure S12. Phosphorescence spectra change of the DMF solution of P3 $\left(\lambda_{\mathrm{ex}}=380 \mathrm{~nm}\right)$ and $\mathbf{5}$ $\left(\lambda_{\mathrm{ex}}=420 \mathrm{~nm}\right)$ before and after UV light irradiation.
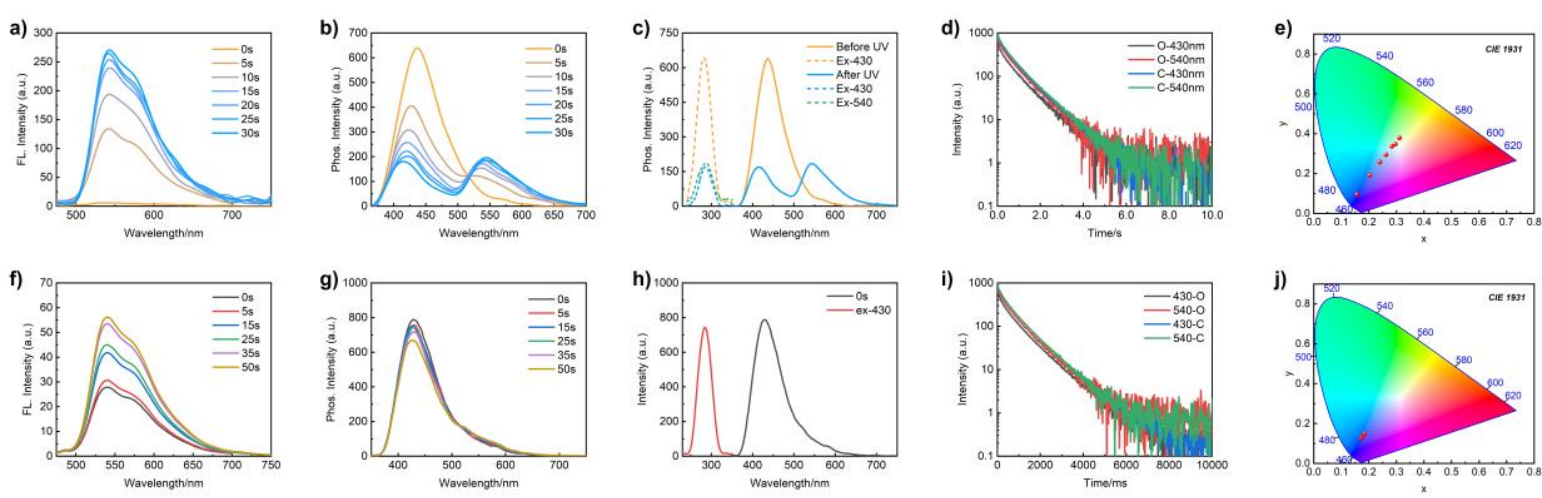

Figure S13. Fluorescence (a, f), phosphorescence (b, g), excitation (c, h), lifetime decay (d, i), and CIE coordinate $(e, j)$ spectra of the DMF solution of P3-5 (up: concentration $=1.0 \times 10^{-4} \mathrm{M}$; down: concentration $\left.=1.0 \times 10^{-5} \mathrm{M}\right)$. 


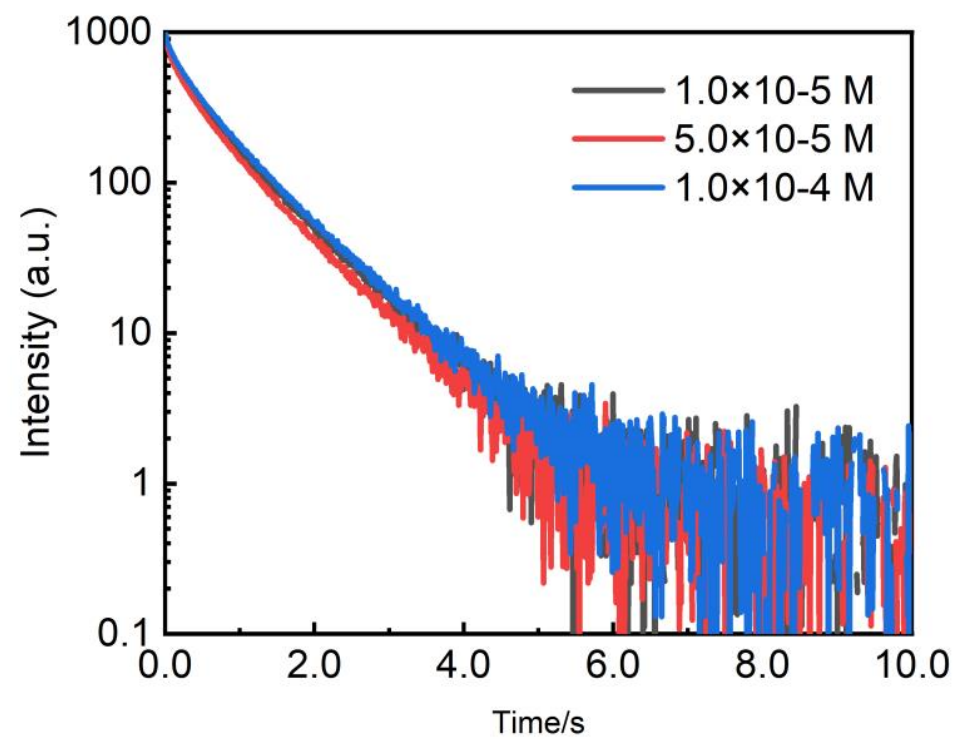

Figure S14. Lifetime decay spectra for the phosphorescence emission at $540 \mathrm{~nm}$ for the DMF solution of P3-5 with different concentrations of $\mathbf{5}$.

\section{Synthesis of the target compounds}

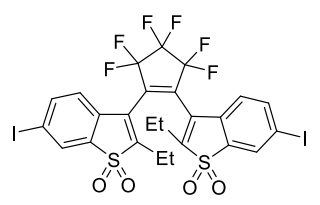

11

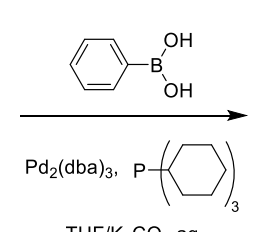

$\mathrm{THF} / \mathrm{K}_{2} \mathrm{CO}_{3}$ aq

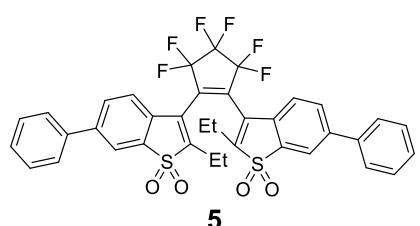

Scheme S2. Synthetic route of compound 5

Compounds 11 and 5 were obtained according to previous report. ${ }^{[1]}$ Saturated aqueous $\mathrm{K}_{2} \mathrm{CO}_{3}$ solution (10 mL), tris(dibenzylideneacetone) dipalladium (0) (30 mg, $0.033 \mathrm{mmol})$, and 18\% tricyclohexylphosphine toluene solution $(0.1 \mathrm{~mL})$ was added to the THF solution of $\mathbf{1 1}$ (150 $\mathrm{mg}, 0.185 \mathrm{mmol})$ and phenylboronic acid $(57 \mathrm{mg}, 0.47 \mathrm{mmol})$. The mixture was stirred at room temperature till the reaction was completed (about $20 \mathrm{~min}$ ). Then the mixture was extracted with ethyl acetate three times. The combined organic phase was evaporated in vacuo, and the residue was purified by silica gel column chromatography (petroleum ether: ethyl acetate $=10$ : 1) to give 5 (119 mg, $0.168 \mathrm{mmol}, 91 \%$ yield) as a white solid. ${ }^{1} \mathrm{H}$ NMR (400 MHz, Chloroform- 
d) $\delta 7.97(\mathrm{~d}, J=1.7 \mathrm{~Hz}, 1.2 \mathrm{H}), 7.90(\mathrm{~d}, J=1.7 \mathrm{~Hz}, 0.8 \mathrm{H}), 7.82(\mathrm{dd}, J=8.0,1.7 \mathrm{~Hz}, 1.2 \mathrm{H}), 7.65$

$-7.57(\mathrm{~m}, 3.3 \mathrm{H}), 7.53-7.37(\mathrm{~m}, 7.8 \mathrm{H}), 7.27(\mathrm{~d}, J=8.4 \mathrm{~Hz}, 1.2 \mathrm{H}), 7.23(\mathrm{~d}, J=8.0 \mathrm{~Hz}, 0.8 \mathrm{H})$,

$2.74-2.35(\mathrm{~m}, 4 \mathrm{H}), 1.43(\mathrm{t}, J=7.6 \mathrm{~Hz}, 2.4 \mathrm{H}), 1.10(\mathrm{t}, J=7.6 \mathrm{~Hz}, 3.6 \mathrm{H})$.

๑

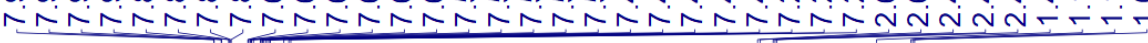

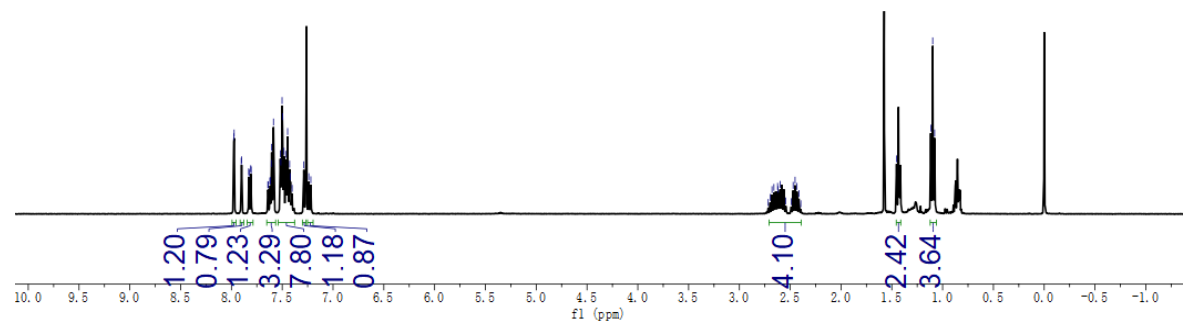

Figure S15. ${ }^{1} \mathrm{H}$ NMR spectra of compound 5.

\section{References}

1. K. Uno, H. Niikura, M. Morimoto, Y. Ishibashi, H. Miyasaka and M. Irie, J. Am. Chem. Soc., 2011, 133, 13558-13564. 